\title{
Uma Experiência sobre a Aplicação de Aprendizagem Baseada em Projetos com Revisão por Pares no Ensino de Gestão de Sistemas de Informação
}

\author{
Leo Natan Paschoal ${ }^{1}$, Simone do Rocio Senger de Souza ${ }^{1}$ \\ ${ }^{1}$ Instituto de Ciências Matemáticas e de Computação (ICMC) - \\ Universidade de São Paulo (USP) \\ Caixa Postal 668 - 13.566-970 - São Carlos - SP - Brasil \\ paschoalln@usp.br, srociodicmc.usp.br
}

\begin{abstract}
Management of Information Systems is a discipline that requires an intensification of intellectual efforts to be taught and learned, because it combines a technical area (Computing) and a social area (Management). In this sense, educational approaches that allow the development of these skills are necessary. This paper presents an experiment report on the use of Project Based Learning and Peer Review during the teaching of this discipline. Therefore, projects that simulate authentic problems that require "real world" solutions have been developed, and an environment that facilitates the development of skills such as autonomy, collaboration and critical thinking has been provided. To evaluate the results, three questionnaires were applied to the students. These questionnaires evaluated the involvement of the students during the practical projects development and the opinion of the students about the used approaches. The results indicate that the used approaches are promising and appropriate to teaching of Management of Information Systems.
\end{abstract}

Resumo. Gestão de Sistemas de Informação é uma disciplina que requer uma intensificação dos esforços intelectuais a serem ensinados e aprendidos, pois combina uma área técnica (Computação) e uma área social (Gestão). Nesse sentido, abordagens educacionais que permitam o desenvolvimento dessas habilidades são necessárias. Este artigo apresenta um relato experimental sobre o uso da Aprendizagem Baseada em Projetos e Revisão por Pares durante o ensino desta disciplina. Portanto, projetos que simulam problemas autênticos que exigem soluções do "mundo real" foram desenvolvidos, e um ambiente que facilita o desenvolvimento de habilidades como autonomia, colaboração e pensamento crítico foi fornecido. Para avaliar os resultados, três questionários foram aplicados aos alunos. Esses questionários avaliaram o envolvimento dos alunos durante o desenvolvimento de projetos práticos e a opinião dos alunos sobre as abordagens utilizadas. Os resultados indicam que as abordagens utilizadas são promissoras e adequadas ao ensino de Gestão de Sistemas de Informação.

\section{Introdução}

O currículo de referência da Sociedade Brasileira da Computação (SBC) menciona que egressos de um curso de Sistemas de Informação devem possuir algumas habilidades, especialmente associadas a resolução de problemas autênticos que fazem parte do "mundo 
real" [Zorzo et al. 2017]. Esses problemas são provenientes de um contexto social ou organizacional e podem ter relação com a identificação de requisitos, desenvolvimento, evolução e/ou administração de Sistemas de Informação. Dentre os componentes curriculares da formação de um Bacharel em Sistemas de Informação, um tópico importante é a Gestão de Sistemas de Informação.

A relevância da Gestão de Sistemas de Informação é observada ao passo que pelo currículo de referência da SBC esse tópico pode ser considerado em um curso de Sistemas de Informação como um eixo temático de formação (Gestão de Sistemas de Informação e da Tecnologia da Informação). O objetivo principal deste eixo é capacitar o aluno em algumas competências tais como: exercer gerência sobre os Sistemas de Informação; aplicar conceitos, vistos durante o curso, adequados à gestão de governança de informação e tecnologia da informação; dentre outros. Além de ser um dos sete eixos temáticos que o currículo da SBC estabelece, o tópico pode ser tratado como um componente curricular do eixo de formação "Desenvolvimento de Software para Sistemas de Informação". Esse componente curricular visa capacitar os estudantes em "avaliar as necessidades de informatizar sistemas, articular visões individuais e organizacionais e apreciar oportunidades de melhorias e ou mudanças em processos, com o uso ou evolução do software" [Zorzo et al. 2017].

Apesar da relevância, ensinar Gestão de Sistemas de Informação é um desafio, pois envolve duas áreas distintas: a Computação, uma área técnica; e a Gestão, uma área social [Devece et al. 2011]. Por envolver essas duas áreas, abordagens de ensino que oportunizam o desenvolvimento de habilidades técnicas e sociais, necessárias para o desempenho das funções que os futuros profissionais irão desempenhar, precisam ser consideradas. [Devece et al. 2011] mencionam sobre a importância de considerar abordagens com foco em promover condições para os estudantes poderem participar ativamente do processo de aprendizagem, como estudos de caso, aprendizagem baseada em problemas e aprendizagem baseada em projetos. Estudo de caso é uma abordagem bastante utilizada na área de Gestão [Cameron et al. 2012]. Em contrapartida, aprendizagem baseada em problemas e aprendizagem baseada em projetos são bastante populares na Computação, especialmente no contexto de desenvolvimento de software [Souza et al. 2016, Fioravanti et al. 2018].

Os currículos atuais de Computação, Nacional e Internacional, representados pela SBC e ACM (Association for Computing Machinery), respectivamente, mencionam a importância dos egressos de cursos de Computação desenvolverem não somente habilidades técnicas, mas também habilidades sociais, as quais são necessárias em atividades de gestão de projetos e de sistemas. Nesse sentido, abordagens ativas de aprendizagem podem ser alternativas viáveis ao ensino de disciplinas como Gestão de Sistemas de Informação. Uma abordagem que visa exercitar o desenvolvimento de habilidades não técnicas como autonomia, colaboração, cooperação e pensamento crítico é a Revisão por Pares (Peer Review) [Kern et al. 2006].

Revisão por Pares é uma abordagem amplamente utilizada no âmbito acadêmico para avaliação de artigos científicos. Ela serve como um meio para o controle da qualidade de artigos científicos que são submetidos a journals e conferências. Seu funcionamento pode ser compreendido da seguinte maneira: quando um artigo é submetido por um pesquisador ou por um grupo de pesquisadores, esse artigo é revisado (avaliado) por 
um grupo de pesquisadores que são especialistas na área. Nesse sentido, a qualidade do artigo é controlada a partir da revisão do mesmo pelos pares de seus pares. No âmbito educacional, essa abordagem vem sendo adaptada, porque pode oferecer diferentes benefícios para os sujeitos que estão envolvidos nos processos de ensino e aprendizagem [Jaime et al. 2016]. Em estudos em que Revisão por Pares é usada como abordagem de apoio ao ensino, os autores adaptam a abordagem para satisfazer os objetivos de aprendizagem que são propostos para uma disciplina e/ou curso [Kern et al. 2003].

Uma característica que se faz presente no uso de Revisão por Pares é a formação de grupos para desenvolvimento de trabalhos. Desse modo, no contexto de uma disciplina, os estudantes são divididos em alguns grupos. Esses grupos são formados com o objetivo de realizar uma atividade de maneira colaborativa, podendo ser uma atividade que resulte em um artigo científico, um relatório ou um projeto. Após a realização da atividade, os trabalhos produzidos são avaliados por estudantes que constituem um outro grupo. Desse modo, cada grupo revisor analisa o trabalho de um outro grupo, seguindo os critérios de revisão que são estipulados pelo professor. Com o uso dessa abordagem, o professor consegue promover a interação e estimular o desenvolvimento do senso crítico dos alunos, fazendo com que os estudantes contribuam para o aprimoramento da qualidade do trabalho desenvolvido por seus pares [Jaime et al. 2016].

Outra abordagem que pode apoiar o aprendizado de estudantes de Gestão de Sistemas de Informação é a aprendizagem baseada em projetos (PBL - Project Based Learning), visto que, essa abordagem oferece subsídios a realização, por parte dos alunos, de atividades que normalmente envolvem projetos que são oriundos do "mundo real". Essa abordagem caracteriza-se pelos seguintes aspectos: os trabalhos são planejados de modo que sejam focados em problemas que levam os alunos a aplicar conceitos e princípios teóricos visto em aula; por meio dos projetos, os alunos são incentivados a desenvolver e/ou exercitar algumas habilidades técnicas e não técnicas; os alunos são envolvidos em atividades autênticas e reais, que normalmente incluem a criação de um produto ou solução, envolvendo tarefas interdisciplinares; a condução do trabalho pode levar semanas e até mesmo meses [Acosta 2016].

Estudos sobre o uso de PBL revelam que a mesma oferece diversos benefícios aos estudantes, promovendo: motivação, condições para os estudantes serem responsáveis pela própria aprendizagem, engajamento na atividade, situações para o aluno experimentar o desenvolvimento de projetos em equipes constituídas por pessoas com diferentes perfis [Jaime et al. 2016, Fioravanti et al. 2018]. Além disso, os currículos de referência de Computação estão recomendando que projetos do "mundo real" sejam explorados no contexto acadêmico, pois é uma maneira de prover condições para os estudantes se prepararem para se inserirem no mercado de trabalho.

Em razão dos benefícios que são oferecidos aos alunos quando as abordagens PBL e Revisão por Pares são utilizadas, [Boubouka and Papanikolaou 2013] mencionam sobre a possibilidade de incorporar Revisão por Pares em PBL. Esse pensamento é posto em prática na disciplina de Gerenciamento de Projetos, conforme relatado no estudo de [Jaime et al. 2016]. Com isso, estudantes formam grupos, desenvolvem os projetos, e após os mesmos serem desenvolvidos, os projetos são revisados por seus pares. Essa mistura de abordagem é capaz de oferecer condições para os estudantes alcançarem um alto nível de sucesso na disciplina [Jaime et al. 2016]. Uma experiência semelhante 
foi realizada no contexto de ensino de Algoritmos e Estruturas de dados, relatada por [Machanick 2005], em que os alunos analisam os projetos uns dos outros.

Diante do que foi exposto, este artigo relata uma experiência conduzida no contexto de uma disciplina de Gestão de Sistemas de Informação, oferecida aos alunos do curso de Bacharelado em Sistemas de Informação, que teve como objetivo principal oferecer condições para que os alunos desenvolvessem diferentes habilidades, não apenas técnicas, durante o aprendizado da disciplina. Essa experiência constitui-se pelo uso de duas diferentes abordagem de apoio ao ensino: PBL e Revisão por Pares. A primeira é usada para instigar os alunos a trabalharem em equipe com projetos do "mundo real", desafiando-os a trabalharem com projetos interdisciplinares que necessitam de apoio colaborativo. Ela também oferece oportunidades para os alunos não somente aprender a teoria da Gestão de Sistemas de Informação, mas aplicar os conceitos vistos durante as aulas em projetos próximos aos que irão desenvolver futuramente no mercado de trabalho. A segunda por sua vez, é utilizada principalmente para oferecer subsídios ao desenvolvimento do senso crítico entre os alunos, e aumentar a qualidade dos projetos desenvolvidos.

Este artigo está estruturado da seguinte maneira. Na seção 2 é descrita a estratégia educacional que foi adotada na disciplina de Gestão de Sistemas de Informação. Na seção 3 são discutidos os resultados obtidos. Por fim, na seção 4 são apresentadas as conclusões do artigo e perspectivas de trabalhos futuros.

\section{Descrição da Experiência}

A experiência da aplicação das abordagens ocorreu na disciplina de Gestão de Sistemas de Informação, oferecida pelo Instituto de Ciências Matemáticas e de Computação da Universidade de São Paulo. Essa disciplina é um componente curricular obrigatório para alunos do curso de Bacharelado em Sistemas de Informação (BSI) e optativo para os cursos de Bacharelado em Ciências de Computação (BCC) e Engenharia de Computação (EngComp). A disciplina é oferecida no sexto semestre para alunos do BSI e pode ser cursada no quinto ano pelos alunos do BCC e EngComp. Para o contexto dessa experiência, a disciplina foi oferecida no $2^{\circ}$ semestre de 2017, possuindo quatro créditos-aula (noventa horas-aula no semestre, divididas em duas aulas semanais), e foi constituída por 31 alunos do BSI, 9 do BCC e 3 do EngComp. O objetivo da disciplina é oferecer uma visão geral sobre as principais aplicações de Sistemas de Informação nas organizações atuais, assim como, os princípios de Governança e Gestão de Serviços de Tecnologia de Informação, focando principalmente na prática que envolve a análise e o dimensionamento de soluções de Sistemas de Informação adequadas ao contexto organizacional.

A experiência foi planejada visando consolidar os conhecimentos que são transpassados no decorrer da disciplina. Para tanto, a disciplina foi planejada de modo que o conteúdo teórico fosse ministrado antes do início do desenvolvimento dos projetos. Assim, ela foi dividida em duas parte. A primeira foi constituída por aulas expositivas sobre o conteúdo, exercícios (intra e extra-classe), estudos de caso, e discussões sobre os exercícios e estudos de casos, visando a participação dos alunos na construção dos conhecimentos teóricos. A segunda parte consistiu no uso da abordagem PBL em conjunto com Revisão por Pares. O foco deste artigo é relatar a experiência realizada na segunda etapa da disciplina, em que os alunos já haviam estudado o conteúdo teórico da disciplina necessário para o desenvolvimento dos projetos. 
Para apoiar a condução da atividade, a disciplina contou com a colaboração de um aluno de mestrado, que desempenhou atividades didáticas em conjunto com o professor da disciplina, na função de estágio docente. Nesse sentido, a estratégia pedagógica que engloba as duas abordagens mencionadas foi planejada da seguinte forma. Inicialmente, o professor da disciplina preparou, em conjunto com o estagiário, três problemas fictícios, que simulam problemas reais. Esses problemas necessitam de soluções que obrigatoriamente envolvem o desenvolvimento de uma solução em formato de projeto. Os problemas foram derivados de outras disciplinas do curso de Sistemas de Informação: em uma disciplina os alunos propuseram esses problemas e em outra foram usados de maneira similar ao que foi feito nesta experiência. Após a definição dos projetos, o professor e o estagiário da disciplina estabeleceram algumas regras para a formação de grupos: (i) os 43 alunos matriculados na disciplina foram convidados a se dividir em 12 grupos; (ii) cada grupo poderia ser constituído por pelo menos 3 e no máximo 4 alunos; (iii) os alunos poderiam definir os grupos. Após a divisão dos grupos, os projetos foram distribuídos randomicamente entre os mesmos, de modo que cada projeto fosse desenvolvido por quatro equipes.

A descrição dos projetos utilizados na disciplina (3 projetos no total) podem ser encontrados no pacote de laboratório que foi preparado para este artigo, disponível em: $<$ https://goo.gl/24CS9t $>$. Em linhas gerais, os grupos teriam que analisar os projetos e propor soluções de Sistemas de Informação adequadas ao contexto da organização em questão. No pacote também foi disponibilizado uma especificação que contém informações sobre o que os alunos deveriam entregar durante o desenvolvimento do projeto. Salienta-se que foi definido que os alunos teriam que fazer duas entregas referentes aos seus projetos, pois a atividade seria desenvolvida em duas fases. Na primeira fase, cada grupo deveria preparar propostas de soluções para automatização do processo das organizações, levando em consideração as especificações. Nessa fase, os alunos deveriam utilizar os conhecimentos adquiridos na disciplina ou em outras relacionadas ao assunto, visando enriquecer as propostas dos projetos. No final desta fase, os alunos deveriam entregar (via ambiente Moodle) e apresentar as soluções elaboradas pelos grupos para todos os colegas da disciplina, ao professor e ao estagiário.

Considerando que 12 grupos foram formados, e cada 4 grupos trabalhou com um projeto diferente, foi determinado que três aulas seriam destinadas a apresentações das soluções propostas pelos grupos, um tema por aula. Salienta-se que cada grupo recebeu a descrição do tema do projeto uma semana antecedente ao prazo para entregar a proposta de solução do projeto. Nesse sentido, cada grupo teve uma semana para desenvolver os projetos. Portanto, no momento em que os projetos foram distribuídos randomicamente entre os grupos, os grupos não tiveram acesso a especificação.

Assim, em uma aula 4 grupos apresentaram suas soluções de projeto para o mesmo tema. Os demais grupos (8) assumiram o papel de revisores dos projetos. Esses grupos receberam as propostas dos grupos para avaliar antes da aula de apresentação. Durante as apresentações, a dinâmica dos seis chapéus foi utilizada para melhorar a participação dos avaliadores no processo.

A dinâmica dos seis chapéus desenvolvida originalmente por Edward De Bono é uma estratégia que visa contribuir com a produção do pensamento crítico, colaboração, comunicação e criatividade [Kwasnik 2014]. Ela fornece diferentes visões para avaliar uma dada situação sobre o ponto de vista único de cada pessoa 
[Scott and Sewchurran 2008]. Utiliza o princípio por trás do pensamento paralelo que prevê que todos os participantes de uma reunião se concentram e pensam no mesmo assunto ao mesmo tempo [Scott and Sewchurran 2008]. De acordo com [Kwasnik 2014] cada um dos seis chapéus representa um espectro do pensamento humano, por exemplo, chapéu azul identifica os benefícios; chapéu vermelho identifica as limitações. Detalhes sobre a dinâmica estão disponíveis no pacote de laboratório.

Para o contexto desta experiência, a dinâmica dos seis chapéus serviu como uma mecanismo de apoio à Revisão por Pares, dado que ao invés de oferecer um formulário para os estudantes preencherem, os mesmos teriam que expressar suas opiniões durante a aula e divulgar a opinião em um fórum de discussão disponibilizado no ambiente virtual de aprendizagem Moodle. Essas opiniões serviram de base para as melhorias das propostas para a segunda fase do projeto. Cada grupo foi sorteado com um chapéu de uma cor e precisou apontar suas percepções sobre o trabalho desenvolvido pelos colegas, considerando a cor do seu chapéu. Ao final de cada apresentação, um avaliador por grupo (de acordo com o chapéu) realizava seus questionamentos e ponderações. A cada apresentação, o chapéu era trocado entre os membros do grupo, de modo que todos que estavam assistindo pudessem colaborar. Durante essa intervenção, o professor e o estagiário ocuparam o papel de mediadores.

Após a avaliação dos alunos em relação às apresentações dos projetos, os alunos avaliadores elegeram a melhor proposta apresentada. Para tanto, deveriam considerar a qualidade de informações presentes no documento entregue e a qualidade da apresentação. Não foi elaborado especificações maiores a respeito de pontos que os alunos deveriam considerar na avaliação. Apenas foi especificado que o documento deveria conter pelo menos as seguintes informações: descrição sobre a empresa, solução proposta (com aspectos técnicos, escopo, melhoria de processo), parâmetro da solução (custo de aquisição, tempo de implantação, treinamento dos profissionais que atuam na organização, infraestrutura necessária, custo operacional), impacto organizacional, impacto social e político. Foi disponibilizado uma enquete no Moodle para que os alunos pudessem eleger a melhor proposta.

Além da avaliação dos seus pares, os grupos receberam um feedback com sugestões de melhorias, emitido pelo professor da disciplina em conjunto com o estagiário. Com as revisões e sugestões de melhorias, a segunda fase foi estabelecida para a melhoria das propostas e reapresentação das mesmas. Semelhantemente a fase anterior, cada 4 grupos responsável por um projeto teria uma aula para fazer a apresentação. Adicionalmente, foi solicitado aos grupos que adicionassem ao documento elaborado os diagramas utilizando BPMN (Business Process Model and Notation) para os processos de negócios, em especial a modelagem do estado original da organização (as is) e a modelagem do estado futuro (to be). Após a entrega do documento e apresentação, os grupos foram novamente avaliados pelos seus colegas da disciplina.

Ao final da experiência os alunos tiveram que responder três questionários. O primeiro questionário foi criado para cada aluno fazer uma autoavaliação sobre a sua participação na atividade. O segundo questionário foi desenvolvido para os alunos avaliarem os seus colegas de equipe, assim, cada aluno avaliou o nível de participação e colaboração dos membros de seus grupos (de maneira anônima). Esses questionários foram construídos para apoiar a avaliação dos trabalhos que foram conduzidos e verificar 
o envolvimento dos alunos no desenvolvimento dos projetos. Por fim, o terceiro questionário teve como objetivo coletar informações sobre a percepção dos alunos quanto às abordagens utilizadas na experiência. Os questionários estão disponíveis no pacote de laboratório. Os resultados serão apresentados na próxima seção.

\section{Resultados Obtidos}

Esta seção apresenta os resultados referentes a essa experiência de ensino, incluindo o envolvimento dos alunos durante as duas fases que englobam o desenvolvimento do projeto, sobre as melhores soluções propostas para os projetos, e sobre a percepção dos mesmos quanto a experiência vivenciada nessa etapa da disciplina.

Para averiguar o envolvimento dos alunos foram utilizados os dois questionário específicos para o envolvimento. Ambos os questionários possuíam as mesmas questões. A única diferença é que um possibilita a autoavaliação e o outro possibilita a avaliação dos pares. Cada questionário possuía 1 questão destinada aos alunos atribuírem uma pontuação para eles mesmos e para seus pares. Sobre a percepção dos alunos na experiência, um questionário foi enviado por $e$-mail para todos os alunos matriculados na disciplina. Apesar da disciplina ter 43 alunos, apenas 40 alunos responderam ao questionário.

Os resultados sobre o envolvimento dos alunos estão resumidos e apresentados na Tabela 1. Nela é possível observar as médias das pontuações por aluno e os desviospadrões, que foram calculados por meio das pontuações que cada aluno recebeu de seus pares em conjunto com a pontuação que cada aluno se atribuiu. Essas pontuações auxiliaram na identificação de alunos que tiveram um maior comprometimento com a equipe e consequentemente um envolvimento maior no desenvolvimento dos projetos. Por exemplo, o aluno número 12, integrante do Grupo 3, ficou com uma média de 3,75 pontos, um valor baixo se considerado o valor máximo que poderia ser obtido.

Tabela 1. Envolvimento dos alunos

\begin{tabular}{|c|c|c|c|c|c|c|c|c|c|c|c|c|c|c|c|}
\hline & \multicolumn{4}{|c|}{ Grupo 1} & \multicolumn{4}{|c|}{ Grupo 2} & \multicolumn{4}{|c|}{ Grupo 3} & \multicolumn{3}{|c|}{ Grupo 4} \\
\hline Aluno & 1 & 2 & 3 & 4 & 5 & 6 & 7 & 8 & 9 & 10 & 11 & 12 & 13 & 14 & 15 \\
\hline Média das pontuações & 9,00 & 9,25 & 9,25 & 4,25 & 7,00 & 8,00 & 7,00 & 8,67 & 7,00 & 4,67 & 9,00 & 3,75 & 5,00 & 5,50 & 8,00 \\
\hline \multirow[t]{2}{*}{ Desvio padrão } & 0,82 & 0,96 & 0,50 & 2,22 & 0,00 & 1,00 & 0,00 & 0,58 & 3,00 & 4,04 & 1,00 & 2,63 & 4,36 & 2,12 & 2,83 \\
\hline & \multicolumn{3}{|c|}{ Grupo 5} & \multicolumn{4}{|c|}{ Grupo 6} & \multicolumn{4}{|c|}{ Grupo 7} & \multicolumn{3}{|c|}{ Grupo 8} & \\
\hline Aluno & 16 & 17 & 18 & 19 & 20 & 21 & 22 & 23 & 24 & 25 & 26 & 27 & 28 & 29 & \\
\hline Média das pontuações & 10,00 & 9,67 & 6,67 & 9,25 & 8,75 & 9,00 & 9,00 & 9,00 & 9,50 & 9,25 & 7,50 & 8,00 & 9,67 & 8,67 & \\
\hline \multirow[t]{2}{*}{ Desvio padrão } & 0,00 & 0,58 & 0,58 & 0,50 & 0,96 & 0,82 & 0,82 & 0,00 & 0,58 & 0,50 & 0,58 & 1,00 & 0,58 & 0,58 & \\
\hline & \multicolumn{3}{|c|}{ Grupo 9} & \multicolumn{4}{|c|}{ Grupo 10} & \multicolumn{3}{|c|}{ Grupo 11} & \multicolumn{4}{|c|}{ Grupo 12} & \\
\hline Aluno & 30 & 31 & 32 & 33 & 34 & 35 & 36 & 37 & 38 & 39 & 40 & 41 & 42 & 43 & \\
\hline Média das pontuações & 9,33 & 9,67 & 9,33 & 8,25 & 8,75 & 9,25 & 7,75 & 7,33 & 7,33 & 10,00 & 8,75 & 9,25 & 9,75 & 9,00 & \\
\hline Desvio padrão & 1,15 & 0,58 & 0,58 & 0,96 & 0,50 & 0,50 & 1,89 & 0,58 & 1,53 & 0,00 & 0,96 & 0,96 & 0,50 & 1,41 & \\
\hline
\end{tabular}

Na mesma tabela também é possível observar os desvios-padrões, que auxiliam na identificação de variações entre as pontuações obtidas pelos alunos. Quanto mais próximo de zero, menor será a diferença entre as pontuações recebidas pelo aluno de seus pares em conjunto com a auto-avaliação. Observando o valor de desvio padrão do aluno número 39, integrante do Grupo 11, nota-se que o mesmo é zero, o que significa que ele se auto avaliou com 10 pontos e toda a sua equipe também atribuiu 10 pontos para ele. Em outras ocasiões é possível observar algumas semelhanças com o que foi mencionado, por exemplo, no Grupo 2, os alunos número 5 e número 7 obtiveram os valores dos desviospadrões equivalentes a zero e a média de ambos os alunos foi 7 , o que demonstra que 
ambos os alunos se autoavaliaram com 7 pontos e os seus pares também os avaliaram com 7 pontos. Nota-se por meio da avaliação a participação efetiva dos alunos no trabalho. Os alunos que se comprometeram foram sinceros e apontaram os alunos que foram passivos na experiência, isto é, que não participaram ativamente no desenvolvimento das soluções.

Conforme mencionado no decorrer deste artigo, os alunos tiveram que escolher as melhores soluções para os projetos. A Figura 1 apresenta os dados das votações e, conforme é possível observar, o grupo que propôs solução para o Projeto 1 melhor votado foi o Grupo 1. O grupo que apresentou a melhor solução para o Projeto 2 foi o Grupo 8. Por fim, o grupo que apresentou a melhor proposta de solução para o Projeto 3 foi o Grupo 12. Os grupos que obtiveram uma melhor votação em relação aos demais foram premiados na disciplina. A ideia da premiação é vista na literatura como positiva, no sentido de trazer elementos relacionados com a motivação, dado que pode fazer com que os alunos fiquem motivados em desenvolver projetos com alta qualidade. Algo semelhante é relatado por [Souza et al. 2016] ao utilizarem a premiação para motivar alunos a desenvolver projetos de software com qualidade. Salienta-se que os alunos foram notificados no início da experiência que as melhores propostas de solução para os projetos seriam premiadas.

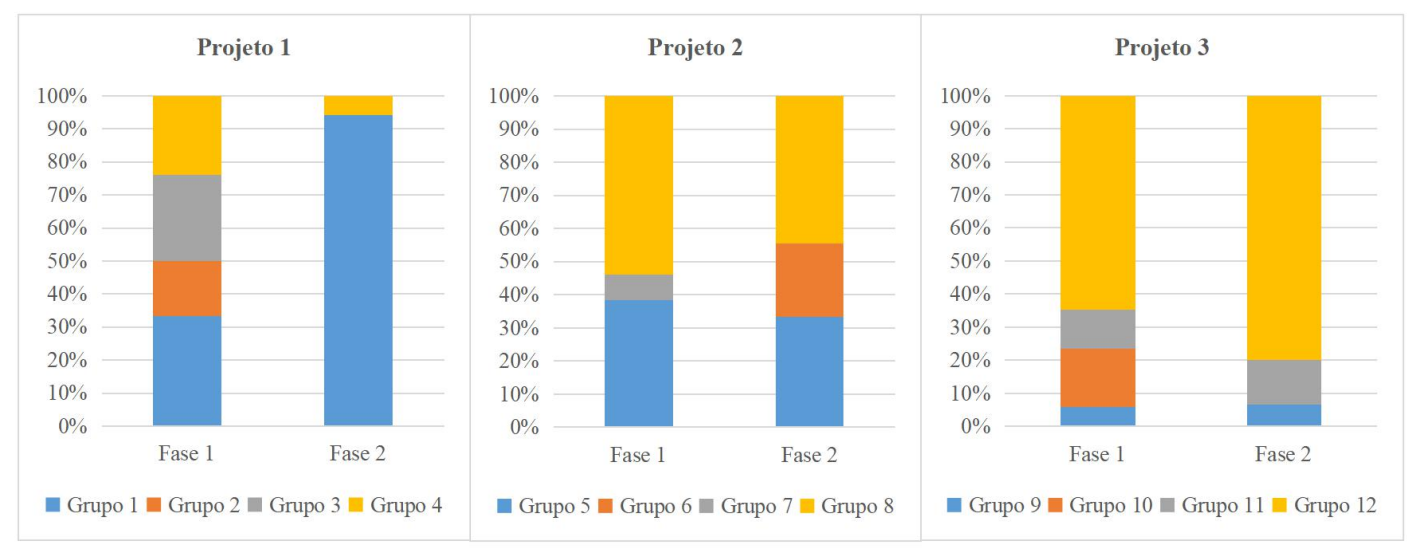

Figura 1. Escolha das melhores propostas de soluções

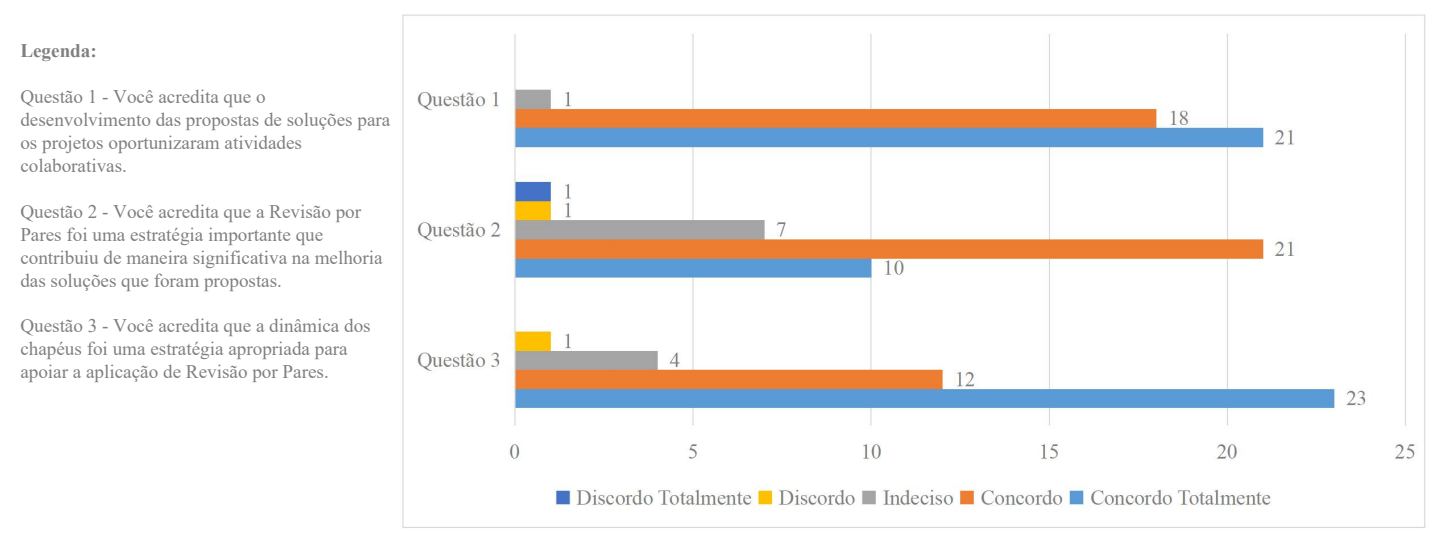

Figura 2. Percepção dos alunos sobre as abordagens

Em relação à percepção dos estudantes sobre a estratégia pedagógica adotada, um terceiro questionário contendo 3 questões fechadas foi disponibilizado. A primeira 
questão teve o intuito de saber se o desenvolvimento do projeto em equipe oportunizou um cenário de atividades que deveriam ser realizadas de forma colaborativa. A maioria dos participantes concordaram com a afirmativa. Aos serem questionados sobre a relevância do uso de Revisão por Pares para melhoria da qualidade dos projetos apresentados, na segunda questão, as respostas oscilaram principalmente entre as opções concordo totalmente, concordo e indeciso. Por fim, quando questionados sobre a importância da dinâmica dos chapéus para apoiar a Revisão por Pares os alunos apontaram que aprovam o uso da dinâmica. A Figura 2 apresenta os resultados. A partir desses dados é possível reconhecer que os alunos aprovaram o uso das abordagens, pois apoiaram a visão prática dos conceitos vistos em aula.

\section{Considerações Finais}

No decorrer deste artigo uma experiência com alunos de Gestão de Sistemas de Informação foi apresentada. Os motivos que levaram o uso das abordagens PBL com Revisão por Pares relacionam-se a capacidade das mesmas oferecerem oportunidades para os alunos trabalharem com projetos que se assemelham ao "mundo real", que poderão ser vistos pelos alunos no mercado de trabalho, com a possibilidade de utilizar a opinião de seus pares visando a melhoria da qualidade do trabalho realizado. A visão mais prática do conteúdo é altamente recomendado pelos currículos de referência da ACM e SBC. Além disso, a experiência buscou contribuir com o desenvolvimento de habilidades não técnicas dos alunos, visto que, as abordagens oferecem subsídios ao trabalho colaborativo, a competição saudável entre os grupos, a importância da comunicação entre os integrantes dos grupos, e a relevância da interpretação dos documentos. Por fim, com o desenvolvimento dos projetos e uso das abordagens esperava-se que os alunos pudessem ter um entendimento em um nível mais prático sobre análise e o dimensionamento de soluções de Sistemas de Informação que são apropriados para as organizações. A partir dos dados apresentados, acredita-se que os objetivos foram atingidos.

Os resultados encontrados com a experiência não visam demonstrar que as abordagens possam ser consideradas as melhores a serem aplicadas para o contexto apresentado, visto que, não foi feito um estudo experimental para averiguar tal condição. Entretanto, eles podem ser utilizados como argumento de que a percepção dos alunos é bastante favorável, podendo inclusive ser vista como uma alternativa para o modelo tradicional de ensino, em que a ênfase do ensino não é a visão mais prática do conteúdo. Em contrapartida, conforme mencionado no decorrer do artigo, o emprego das abordagens criou condições para capacitar os alunos a aprender o conteúdo com um nível maior de profundidade. Assim, espera-se que essa experiência seja replicada por outros professores da área, de forma a contribuir com a formação dos alunos.

\section{Agradecimentos}

Os autores gostariam de agradecer ao CNPq, CAPES e a Fundação de Amparo à Pesquisa do Estado de São Paulo (FAPESP - Processo 2017/10941-8) pelo apoio financeiro.

\section{Referências}

Acosta, O. C. (2016). Recomendação de conteúdo em um ambiente colaborativo de aprendizagem baseada em projetos. $\mathrm{PhD}$ thesis, Centro de Estudos Interdisciplina- 
res em Novas Tecnologias da Educação, Universidade Federal do Rio Grande do Sul, Porto Alegre, Rio Grande do Sul, Brasil.

Boubouka, M. and Papanikolaou, K. A. (2013). Alternative assessment methods in technology enhanced project-based learning. International Journal of Learning Techno$\log y, 8(3): 263-296$.

Cameron, A.-F., Trudel, M.-C., Titah, R., and Léger, P.-M. (2012). The live teaching case: a new is method and its application. Journal of Information Technology Education: Research, 11:27-42.

Devece, C., Lapiedra, R., and Gil, I. (2011). Teaching information systems in business management studies: Basic competencies to achieve and methodologies assessment. In 5th International Multi-Conference on Society, Cybernetics and Informatics, pages 114-119.

Fioravanti, M. L., Sena, B., Paschoal, L. N., Silva, L. R., Allian, A. P., Nakagawa, E. Y., Souza, S. R., Isotani, S., and Barbosa, E. F. (2018). Integrating project based learning and project management for software engineering teaching: An experience report. In 49th ACM Technical Symposium on Computer Science Education, pages 806-811.

Jaime, A., Blanco, J. M., Domínguez, C., Sánchez, A., Heras, J., and Usandizaga, I. (2016). Spiral and project-based learning with peer assessment in a computer science project management course. Journal of Science Education and Technology, 25(3):439449.

Kern, V., dos Santos Pacheco, R., Saraiva, L., and Pernigotti, J. (2006). Peer review in computer science: Toward a regular, large scale educational approach, chapter 3, pages 45-65. Information Science Publishing, EUA.

Kern, V. M., Saraiva, L. M., and dos Santos Pacheco, R. C. (2003). Peer review in education: Promoting collaboration, written expression, critical thinking, and professional responsibility. Education and Information Technologies, 8(1):37-46.

Kwasnik, M. (2014). Nature of creativity in computer science education. designing innovative workshops for cs students. In International Conference on Multimedia, Interaction, Design and Innovation, pages 8:1-8:7.

Machanick, P. (2005). Peer assessment for action learning of data structures and algorithms. In 7th Australasian Conference on Computing Education, pages 73-82.

Scott, E. and Sewchurran, K. (2008). Reflection-in-action: Using experience to reconstruct meaning in a learning environment. In International Conference on Computer Science and Software Engineering, pages 81-86.

Souza, S. R. S., Oliveira, B. H., Grillo, F., and Cico, C. (2016). Construção de plataformas digitais durante o ensino de engenharia de software: um relato de experiência. In $I X$ Fórum de Educação em Engenharia de Software (FEES 2016), pages 13-22.

Zorzo, A. F., Nunes, D., Matos, E. S., Steinmacher, I., Leite, J. C., Araujo, R., Correia, R. C. M., and Martins, S. (2017). Referenciais de formação para os cursos de graduação em computação 2017. Technical report, Porto Alegre, Brasil. 